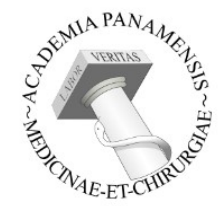

Cerrud-Rodriguez y colaboradores.

Lecciones desde el epicentro de la pandemia.

Título: Lecciones desde el epicentro occidental de la pandemia: la experiencia de Montefiore Health System en el manejo de pacientes infectados con COVID-19 en la ciudad de Nueva York

Title: Lessons learned from the pandemic's Western epicenter: the experience of Montefiore Health System in the management of patients infected with COVID-19 in New York City

Autores: Cerrud-Rodriguez, Roberto ; Victoria, Gerardo ${ }^{* *}$; Sandoval, Julio ${ }^{* * *}$; Cuero, César $^{* * * *}$; Alfaro, Fernando ${ }^{* * * *}$; García, Mario J.

*. División de Cardiología, Montefiore Health System/Albert Einstein College of Medicine. Ciudad de Nueva York. Estados Unidos.

**. Hospital Santo Tomás. Ministerio de Salud. Ciudad de Panamá. República de Panamá. ***. Comité Asesor del Ministerio de Salud en asuntos relacionados a la pandemia de COVID-19. República de Panamá.

****. Caja de Seguro Social. Ciudad de Panamá. República de Panamá.

Palabras clave: COVID-19, pandemia, hipercoagulabilidad, tormenta de citoquinas Keywords: COVID-19, pandemic, hypercoagulability, cytokine storm

Correspondencia a: Dr. Roberto C. Cerrud-Rodriguez. Montefiore-Einstein Center for Heart and Vascular Care, Montefiore Health System, 111E $210^{\text {th }}$ St, Foreman Pavilion, Bronx, NY 10467, Estados Unidos. Email: robertocerrud@gmail.com Teléfono: +1-718920-7000

Declaración de conflicto de intereses: Los autores declaran que no existe conflicto de interés alguno asociado en la publicación de este manuscrito.

\title{
Resumen
}

Montefiore Health System, red de hospitales universitarios afiliados al Albert Einstein College of Medicine, ha sido uno de los principales frentes de batalla contra el COVID-19 en la ciudad de Nueva York, epicentro occidental de la pandemia. Con múltiples campuses localizados principalmente en el Bronx, donde la tasa de infección por COVID-19 es de 2,048/100,000 habitantes, ha tenido que enfrentar la pandemia desde sus inicios. La experiencia acumulada a lo largo de estos meses es invaluable, aunque desafortunadamente gran parte de ella ha sido adquirida en base a ensayo y error, dada la novedad de este virus y la ausencia de guías de manejo basadas en la evidencia. Nuestra experiencia es también relevante ya que el $35 \%$ de nuestra población es de raza Hispana. En las siguientes páginas repasamos aquello que sabemos sobre los inicios del SARS-CoV-2, las tesis más aceptadas

Publicación de la Revista Médica de Panamá. https://www.revistamedica.org

Manuscrito recibido: 23 de abril ,2020. Fecha de publicación temprana: 24 de abril, 2020.

DOI: http://dx.doi.org/10.37980/im.journal.rmdp.2020881

Editorial Infomedic International https://www.infomedicint.com

Acceso libre. Para uso individual; no comercial. Derechos reservados. 


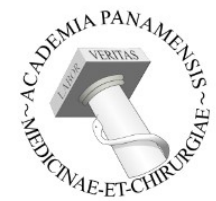

acerca de su fisiopatología, su curso clínico, el manejo inicial una vez el paciente es admitido a nuestra institución con el diagnóstico de COVID-19. También exponemos nuestras experiencias en el manejo terapéutico de las complicaciones multiorgánicas que provoca esta entidad.

\begin{abstract}
Montefiore Health System, a network of university hospitals affiliated with the Albert Einstein College of Medicine, has been one of the main fronts in the battle against COVID19 in New York City, western epicenter of the pandemic. With multiple campuses located mainly in The Bronx, where the rate of infection is of 2,048/100,000 inhabitants, it had to fight the pandemic since its very beginning. The experience gained during these past months is invaluable, although unfortunately most of it had to be learned by trial and error, given the novelty of this coronavirus and the complete lack of evidence-based guidelines. Our experience is particularly relevant given that $35 \%$ of our patient population is of Hispanic heritage. In the next few pages, we'll go over what we currently know about the beginnings of SARS-CoV-2, the currently accepted hypothesis about its pathogenesis, its clinical course, and its initial management once a COVID+ patient is admitted to our institution. We also share our experiences with the clinical management of the multiorgan complications caused by this disease.
\end{abstract}

\title{
Introducción
}

Los primeros reportes de una nueva infección viral en la ciudad de Wuhan, provincia de Hubei, República Popular China fueron realizados en diciembre de 2019. Para el 26 de enero de 2020, ya había 2794 casos confirmados y 80 muertes reportadas (1). Esta enfermedad, cuyo agente etiológico recibió el nombre formal de SARS-CoV-2 por el Comité Internacional de Taxonomía Viral, provoca la enfermedad ahora conocida como COVID-19, responsable de la primera pandemia del siglo XXI. Es hecho harto conocido el nivel de disrupción a nivel de sistemas de salud, de la economía y del tejido social que ha provocado esta pandemia a una escala global.

Mediante el siguiente escrito, los autores esperan poder divulgar la experiencia obtenida durante el manejo intrahospitalario de miles de pacientes afectados por la pandemia de COVID-19 en la ciudad de Nueva York, epicentro occidental de esta enfermedad.

Montefiore Health System, red de hospitales universitarios afiliados al Albert Einstein College of Medicine, ha sido uno de los principales frentes de batalla contra el COVID-19 en la ciudad de Nueva York, epicentro occidental de la pandemia. Con múltiples campus localizados principalmente en el Bronx, donde la tasa de infección por COVID-19 es de 2,048/100,000 habitantes, ha tenido que enfrentar la pandemia desde sus inicios, manejando números astronómicos de pacientes admitidos por esta enfermedad: 2013 pacientes admitidos en un mismo periodo tiempo, con un máximo 476 pacientes con COVID-19 admitidos a las unidades de cuidados intensivos (Figura $\mathrm{N}^{\mathrm{o}} 1$ ). Nuestro deseo es poder 


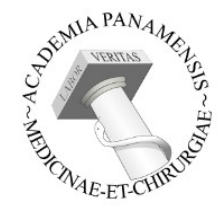

ofrecer nuestra experiencia en el manejo de los pacientes críticos hospitalizados a la América Latina, en especial a la República de Panamá como orientación, tomando en cuenta la falta de guías de manejo basadas en la evidencia, al ser este una nueva enfermedad nunca antes vista en la historia humana.

Es nuestro más sincero deseo que nuestra experiencia le permita a América Latina sortear con el mayor éxito y la menor pérdida de vidas posible el azote de esta pandemia y que sea visto como una oportunidad de mejorar las guías locales existentes, tomando siempre en cuenta que esta publicación se basa principalmente en la experiencia de un centro con un muy alto volumen de casos, y no de guías realizadas con la rigurosidad estadística que una época normal permitiría.

\section{Fisiopatología del COVID-19}

Antes de iniciar esta sección debemos resaltar que lo que conocemos acerca de la fisiopatología del SARS-CoV-2 hasta el día de hoy, cambia minuto a minuto. Mucho de lo que pensábamos saber en un inicio acerca de este nuevo coronavirus ha sido puesto en tela de juicio por la comunidad científica internacional debido a la inconsistencia en los primeros reportes publicados en China (2).

El mecanismo mediante el cual el SARS-CoV-2 produce la enfermedad aún no se ha elucidado de manera definitiva, pero una de las tesis más aceptadas señala que tiene que ver con las proteínas de superficie capaces de ligarse al receptor ACE2 y de esta forma infectar las células del epitelio pulmonar e intestinal (3). El receptor de superficie ACE2 también se encuentra presente en las células endoteliales arteriales y venosas y en las células de músculo liso de múltiples órganos (4).

En autopsias realizadas en pacientes chinos, tanto en fase temprana como tardía de COVID19, se demostró daño alveolar difuso con formación de membrana hialina, infiltración de células mononucleares y macrófagos en el alveolo, y engrosamiento difuso de la pared alveolar. Adicionalmente se encontró hemorragia renal, inflamación hepática, atrofia esplénica y necrosis linfática (5). En una serie de autopsias realizadas en Brasil por Dohlnikoff y colaboradores, se encontró lesiones similares en los pulmones, además de un número variable de trombos fibrinosos en las arteriolas pulmonares en 8 de 10 autopsias realizadas (6).

La infección por SARS-CoV-2 lleva a desarrollar una tormenta de citoquinas mediada por una desregulación inmune que produce una excesiva liberación de factor de necrosis tumoral alfa, interleukina-1 $\beta$, IL-6, GSF, proteína 10 inducida por interferón gamma, así como proteínas inflamatorias de monocitos y macrófagos (5).

Un grupo italiano, liderado por Ciceri y colaboradores, va más allá, y postula que, en individuos predispuestos, el SARS-CoV-2 no sólo provoca daño directo por la infección

Publicación de la Revista Médica de Panamá. https://www.revistamedica.org

Manuscrito recibido: 23 de abril ,2020. Fecha de publicación temprana: 24 de abril, 2020.

DOI: http://dx.doi.org/10.37980/im.journal.rmdp.2020881

Editorial Infomedic International https://www.infomedicint.com

Acceso libre. Para uso individual; no comercial. Derechos reservados. 


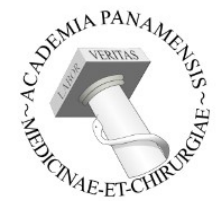

viral, sino que esta desregulación inmune provoca un estado hiperinflamatorio con activación del complemento, provocando daño difuso masivo del endotelio vascular pulmonar, lesiones severas al epitelio alveolar y trombosis microvascular (4). Clínicamente, esto se manifiesta con un empeoramiento progresivo del desbalance ventilación/perfusión con pérdida del reflejo de vasoconstricción pulmonar en respuesta a la hipoxia y eventual diseminación de la microtrombosis local de los pulmones a otros órganos como los riñones, el corazón y el cerebro (4).

\section{Curso Clínico del COVID-19}

El COVID-19 tiene un curso clínico prolongado y, según datos de los Centros de Control de Enfermedades de China, puede llegar a ser asintomática o pauci-asintomática en el 81\% de los casos, severa (disnea, hipoxia o $>50 \%$ de involucramiento pulmonar en estudio de imágenes del tórax) en el 14\% y crítica (falla respiratoria, shock o falla multiorgánica) en el $5 \%$ de los casos (7). La mortalidad global es de aproximadamente el $2 \%$, y se da más en hombres y en pacientes de la tercera edad. La hipertensión y la diabetes parecen estar asociados a un mayor riesgo de morir $(7,8)$.

El COVID-19 tiene un período de incubación promedio de 14 días, en algunos casos tiene manifestaciones clínicas tan temprano como 4 o 5 días luego de ser expuestos (9). La duración promedio de la capacidad de diseminar el virus es de 20 días, aunque ha habido casos en los que se ha detectado incluso hasta los 37 días en supervivientes y hasta el momento de la muerte en aquellos que sucumben ante la enfermedad (10).

Los pacientes que son admitidos y necesitan intubación con ventilación mecánica pueden permanecer intubados por varias semanas. No existen datos sólidos acerca de la supervivencia de pacientes con COVID-19 severo que sufren paro cardíaco, pero de forma anecdótica el porcentaje de pacientes que logran recuperar circulación espontánea es extremadamente bajo (11).

\section{Estrategias de Prevención}

No existe en la actualidad ninguna vacuna que prevenga la infección por COVID-19. El lavado de manos utilizando agua y jabón antibacterial por al menos 20 segundos, el uso de gel alcoholado como alternativa para desinfectarse las manos, las técnicas de distanciamiento físico como mantenerse a 1.5 metros de distancia de otras personas al salir en público, evitar salir del domicilio a menos que se trate de situaciones ineludibles y, desde hace unos pocos días, el uso obligatorio de mascarillas quirúrgicas, o hechas de tela, en público, han sido utilizados como medidas para tratar de disminuir la tasa de contagio del COVID-19 en la ciudad de Nueva York y evitar el colapso total del sistema sanitario. Cabe resaltar que se ha demostrado que el SARS-CoV-2 se transmite por contacto directo con superficies contaminadas, por aerosol e, incluso, bajo ciertas circunstancias, puede transmitirse por vía aérea directa (12). 


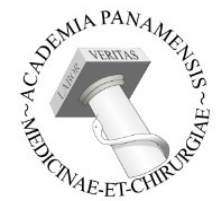

En el ámbito intrahospitalario, el gobierno del Estado de Nueva York ha prohibido la visita a todos los pacientes con excepción de las pacientes que se encuentren en labor de parto (a las que se permite un acompañante) y de los pacientes pediátricos (a los que se permite ser acompañados por sus padres). En cuanto al personal sanitario de la institución, debe utilizar mascarillas quirúrgicas en todo momento. En los momentos en que el personal de salud se haga cargo de pacientes bajo investigación por COVID-19 o de pacientes con casos confirmados de COVID-19 o se encuentre en una de las salas de COVID-19, debe utilizar estrictamente el equipo de protección personal adecuado: mascarillas N95, escudo facial, bata desechable, guantes desechables y gorro quirúrgico desechable, con el fin de disminuir al mínimo la posibilidad de infección.

\section{Manejo de los Pacientes Hospitalizados}

Los pacientes admitidos bajo sospecha de COVID-19 o con un caso confirmado de la enfermedad deben ser monitorizados de manera constante por signos de deterioro de su función respiratoria. Una saturación de oxígeno $<94 \%$ sin oxígeno suplementario debe llevar a una respuesta escalada, rápida y agresiva: oxígeno por cánula nasal, mascarilla con reservorio, cánula nasal de alto flujo e intubación endotraqueal con ventilación mecánica. La utilización de ventilación de presión positiva no-invasiva (CPAP, BiPAP) no es recomendada por la generación de grandes cantidades de aerosol (13).

Las intubaciones endotraqueales de pacientes con COVID-19 deben ser realizadas por el personal más experto y rápidamente disponible en la institución (medicina crítica, anestesiología) utilizando el equipo de protección personal ya descrito y colocando una barrera física para disminuir el contacto directo con el paciente. En nuestra institución, los pacientes con COVID-19 que van a ser intubados son cubiertos con una sábana de vinil de 6 pies de ancho por 9 pies de largo y de un grosor de al menos $1 \mathrm{~mm}$, con un par de agujeros (con una distancia de 18 pulgadas entre sí) al nivel de la cabeza del paciente para las manos de quien vaya a realizar la intubación y otros agujeros laterales para las manos de los asistentes (Figura No2).

En caso de intubación endotraqueal prolongada, se prefiere evitar la realización de traqueostomías dado el elevado riesgo de generación de aerosol no solamente durante el procedimiento, sino también durante el cuidado diario de la traqueostomía. Hay que recordar también que entre mayor sea el tiempo que un paciente con COVID-19 permanezca intubado, mayor la mortalidad. Según cifras oficiales del Departamento de Salud del Estado de Nueva York, la mortalidad de los pacientes con COVID-19 que llegan a necesitar requerir una intubación prolongada ronda el $88.1 \%$ (11). También se desaconseja la realización de broncoscopías en pacientes con COVID-19 debido al alto riesgo de generación de aerosoles e infección al personal de salud.

Además de los exámenes de laboratorio y de gabinete usuales (hemograma completo con diferencial, química sérica, creatinina, BUN, perfil de coagulación, LDH, urinálisis, rayos

Publicación de la Revista Médica de Panamá. https://www.revistamedica.org

Manuscrito recibido: 23 de abril ,2020. Fecha de publicación temprana: 24 de abril, 2020.

DOI: http://dx.doi.org/10.37980/im.journal.rmdp.2020881

Editorial Infomedic International https://www.infomedicint.com

Acceso libre. Para uso individual; no comercial. Derechos reservados. 


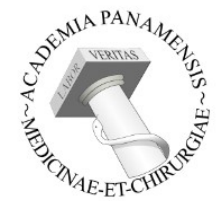

X de tórax), para los pacientes con COVID-19, añadimos la siguiente batería de exámenes: niveles de ferritina sérica, BNP, troponina T, IL-6, proteína C-reactiva, Dímero-D, procalcitonina y lactato sérico. La tomografía de tórax, aunque es útil, no se utiliza rutinariamente debido a la potencial contaminación de los equipos y al hecho que ya contamos con una prueba de reacción en cadena de polimerasa de SARS-CoV-2 mediante hisopado orofaríngeo y nasofaríngeo que podemos realizar dentro de nuestro propio laboratorio y nos da resultados en cuestión de horas.

Dentro de los laboratorios rutinarios, la linfopenia, la transaminitis, una LDH elevada, alteraciones en el perfil de coagulación y falla renal aguda están asociadas a un peor pronóstico (14).

Los niveles de ferritina sérica y proteína C-reactiva suelen estar elevados y los utilizamos como indicación temprana para el uso de esteroides. La procalcitonina casi siempre se encuentra normal, a menos que haya sobreinfección bacteriana. Niveles de dímero $\mathrm{D} \geq 2$ $\mathrm{mcg} / \mathrm{mL}$ son utilizados en Montefiore Health System para comenzar anticoagulación sistémica, como describimos más adelante. Los niveles de troponina $\mathrm{T}$ elevados están asociados a mayor riesgo de mortalidad intrahospitalaria (15), aunque aún no está claro cómo se puede modificar este factor de riesgo, más allá de monitorizar por arritmias y signos de colapso cardiovascular. Niveles de BNP elevados "de novo” pueden indicar falla cardiaca mediada por COVID-19; en caso de shock cardiogénico, se puede utilizar dobutamina (inotrópico) o milrinona (inodilatador) como agentes de soporte. En caso de shock séptico, luego de la resucitación inicial, se inicia norepinefrina como vasopresor de primera línea.

La IL-6 la utilizamos como marcador debido a múltiples estudios randomizados que se están llevando a cabo dentro de nuestra institución con drogas como el sarilumab (anticuerpo monoclonal anti-receptor de IL-6). También somos parte del estudio de remdesivir (antiviral directo), del tocilizumab (anticuerpo monoclonal anti-receptor de IL6) y del estudio federal sobre la potencial efectividad de la hidroxicloroquina. Dado que estos son estudios en curso, cuya efectividad no ha sido demostrada y son propiedad intelectual privada, no podemos proveer mayor información sobre los mismos. La excepción a esto es la hidroxicloroquina, que ya existe como genérico desde hace décadas y ha sido tradicionalmente utilizado como antimalárico y antirreumático.

La hidroxicloroquina, la utilizamos de la siguiente forma: $400 \mathrm{mg}$ BID el día 1, y luego 400 mg diarios por un total de 5-10 días, dependiendo de la severidad clínica que demuestre el paciente. Se debe monitorizar el QTc de estos pacientes de forma regular y el tratamiento debe suspenderse si el mismo sobrepasa los $500 \mathrm{~ms}$. Sin embargo, resultados preliminares de un análisis retrospectivo publicado por Magagnoli y colaboradores utilizando la base de datos del sistema de hospitales de veteranos de Estados Unidos (Veterans Administration, VA) que analizó el curso clínico de 368 pacientes hospitalizados con COVID-19, divididos

Publicación de la Revista Médica de Panamá. https://www.revistamedica.org

Manuscrito recibido: 23 de abril ,2020. Fecha de publicación temprana: 24 de abril, 2020.

DOI: http://dx.doi.org/10.37980/im.journal.rmdp.2020881

Editorial Infomedic International https://www.infomedicint.com

Acceso libre. Para uso individual; no comercial. Derechos reservados. 


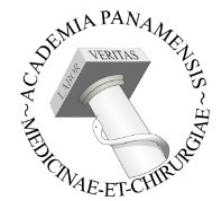

en grupos de pacientes que no recibieron ni hidroxicloroquina (HCQ) ni azitromicina $(n=158)$, aquellos que recibieron HCQ solamente $(n=97)$ y aquellos que recibieron una combinación de HCQ con azitromicina $(n=113)$ (16). No se encontró evidencia que el uso de HCQ, con o sin azitromicina, disminuya el riesgo de intubación y ventilación mecánica en comparación con el grupo de pacientes que no recibió ninguno de estos medicamentos; además, en el grupo que recibió HCQ solamente, hubo un riesgo significativamente mayor de mortalidad en comparación con la cohorte que no recibió ningún medicamento (HR ajustado 2.61; 95\% CI 1.10-6.17, p=0.03). En el grupo de pacientes que recibió HCQ en conjunto con azitromicina, hubo una tendencia a mayor mortalidad en comparación con el grupo que no recibió ningún medicamento (HR ajustado 1.14; 95\% CI 0.56-2.32, p=0.72) (16). Cabe destacar que al ser este un estudio pequeño y retrospectivo, debemos esperar se confirmen sus resultados con los estudios prospectivos randomizados que se encuentran actualmente en ejecución. No hay rol rutinario en nuestra institución para el manejo de COVID-19 con azitromicina, ni con vitamina $C$, ni con vitamina $D$. El uso concomitante de hidroxicloroquina con azitromicina en las primeras semanas de la pandemia sólo resultó en un aumento en la incidencia de arritmias ventriculares sin obtener ningún beneficio para los pacientes y el ácido ascórbico es utilizado como placebo en un estudio clínico en curso, iniciado por la University of Washington, tratando de elucidar si la hidroxicloroquina se puede utilizar como profiláctico contra el COVID-19 en personas con historia de exposición de alto riesgo (ClinicalTrials.gov Identifier: NCT04328961).

\section{Soporte con Ventilación Mecánica}

Utilizamos el protocolo ARDSNet, procurando utilizar volumen tidales bajos y PEEP altos para tratar de mantener la saturación de oxigeno entre 88\%-92\% (17). En pacientes que aun no han requerido intubación, se les solicita que se coloquen en posición prona para dormir y se les administra oxígeno suplementario de acuerdo con su requerimiento individual (cánula nasal, cánula nasal de flujo alto). En pacientes intubados y mecánicamente ventilados que no responden de manera adecuada (usualmente definido por un PF ratio $<150$ ), los colocamos en posición prona durante 16 horas al día luego de asegurarnos que están perfectamente sedados. En caso de disincronía con el ventilador, se utiliza sedación y paralíticos. Hemos visto caso de respuesta paradójica donde el pronar al paciente empeora la oxigenación y la saturación de oxígeno cae por debajo de $88 \%$ - si observan este fenómeno, asuman que se trata de un tromboembolismo pulmonar y utilicen inhibidores directos de la trombina para su manejo, como explicaremos mas adelante.

\section{Manejo Clínico de la Tormenta de Citoquinas y del estado de Hipercoagulabilidad}

Nuestra estrategia de manejo se basa en dos principios fundamentales: controlar la tormenta de citoquinas de forma temprana y evitar los eventos tromboembólicos que caracterizan esta enfermedad.

La tormenta de citoquinas la manejamos de una forma similar a la que se sugiere en las guías locales panameñas, utilizando esteroides por 5 días y guiándonos para su utilización

Publicación de la Revista Médica de Panamá. https://www.revistamedica.org

Manuscrito recibido: 23 de abril ,2020. Fecha de publicación temprana: 24 de abril, 2020.

DOI: http://dx.doi.org/10.37980/im.journal.rmdp.2020881

Editorial Infomedic International https://www.infomedicint.com

Acceso libre. Para uso individual; no comercial. Derechos reservados. 


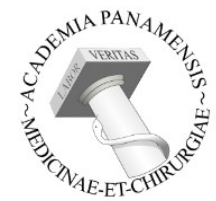

por marcadores séricos de inflamación, particularmente la proteína C-reactiva. Para pacientes no intubados usamos prednisona $1 \mathrm{mg} / \mathrm{kg}$ como bolo en día 1 , mientras que los días 4 a 5 dividimos la dosis total en una administración BID. Para pacientes intubados y mecánicamente ventilados, el manejo es similar, sólo que preferimos utilizar metilprednisolona intravenosa, también en dosis de $1 \mathrm{mg} / \mathrm{kg}$ y administrada de la misma forma, por el mismo período de tiempo. Esta última está asociada a una disminución de la mortalidad en pacientes con distrés respiratorio agudo debido a COVID-19 en un estudio publicado por $\mathrm{Wu}$ y colaboradores (14).

Hemos observado, como evidencia anecdótica en nuestra institución y en muchas otras en la ciudad de Nueva York, que muchas veces la causa de muerte de los pacientes con COVID-19 es debida a embolismo pulmonar, incluso en personas jóvenes y sin factores de riesgo clásicos. En estudios realizados en Holanda y recientemente publicados, se ha encontrado que existe pacientes en los que el COVID-19 provoca resistencia a la heparina y productos derivados, debido a un aumento desproporcionado del factor VIII, fibrinógeno y dímero-D (18). Estos niveles anormalmente elevados de fibrinógeno, hace a los pacientes de COVID-19 resistentes de manera intrínseca a la heparina incluso a niveles terapéuticos, además que el hecho de que muchos de estos pacientes entren en falla renal aguda, lo que impide la utilización de heparina de bajo peso molecular (19). También tenemos que considerar el hecho que la necesidad de monitorizar el PTT cada 6 horas en caso de goteo de heparina no fraccionada aumenta el riesgo de exposición del personal. En base a estos datos y a nuestra propia experiencia clínica con estos pacientes, nuestro departamento de Hematología y Trombosis diseñó un algoritmo, en el que a los pacientes hospitalizados en sala con síntomas moderados de COVID-19 se les inicia anticoagulación con apixaban 5 mg dos veces al día. Mientras tanto, aquellos pacientes en falla respiratoria aguda que requieren intubación, ventilación mecánica y admisión a sala de cuidados intensivos, se les inicia en un goteo de inhibidores directos de la trombina, ya sea argatroban o bivalirudina, dado que tuvimos casos de trombosis venosa profunda y sospecha de tromboembolismo pulmonar durante las primeras semanas de la pandemia, en las que usábamos apixaban en este grupo de pacientes críticamente enfermos. No existe un período de tiempo predeterminado en el cual mantengamos a ambos grupos de pacientes anticoagulados. La práctica común es mantener a los pacientes intubados en bivalirudina hasta varios días después de ser extubados y cuando haya una relativa estabilización de la función respiratoria, en cuyo momento se suspende la infusión de bivalirudina y se inicia apixaban oral. Este último se mantiene hasta 3 días después de haber sido dado de alta el paciente.

\section{Manifestaciones cardíacas del COVID-19}

Mediante un mecanismo no elucidado aún, hemos notado que el COVID-19 provoca cuadros de miocarditis que simulan perfectamente infartos al miocardio con elevación del segmento ST (IAMEST) con dolor precordial, elevaciones en el segmento ST, elevación de troponina sérica (Figura No3). Es por esta razón que no recomendamos la utilización de trombolíticos en la medida que las instituciones tengan la capacidad de realizar

Publicación de la Revista Médica de Panamá. https://www.revistamedica.org

Manuscrito recibido: 23 de abril ,2020. Fecha de publicación temprana: 24 de abril, 2020.

DOI: http://dx.doi.org/10.37980/im.journal.rmdp.2020881

Editorial Infomedic International https://www.infomedicint.com

Acceso libre. Para uso individual; no comercial. Derechos reservados. 


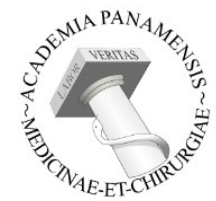

cateterismos cardiacos con las medidas de bioseguridad adecuadas. Un luminograma no obstructivo efectivamente elimina al IAMEST del diagnóstico diferencial y permite enfocar el tratamiento en medidas de soporte hemodinámico si el paciente así lo amerita. Aunque no existe aún tratamiento que haya demostrado éxito en el manejo de la miocarditis por COVID-19, en ciertos pacientes se ha utilizado inmunoglobulina intravenosa como medida de último recurso. No existe evidencia que el levosimendan sea de utilidad para el manejo de esta entidad.

\section{Manifestaciones renales del COVID-19}

El COVID-19 puede provocar cuadros de falla renal aguda severa, con oliguria o anuria, que puede llegar a requerir hemodiálisis continua veno-venosa. En nuestra experiencia, es recomendable involucrar a los equipos de nefrología de forma temprana al ser detectada esta entidad, que trae consigo un mal pronóstico. En algunos casos hemos observado que la creatinina tiende a regresar lentamente a la normalidad al ser tratados por varios días con una infusión continua de bivalirudina. Es también destacable que, antes de que se generalizara el uso de la bivalirudina en nuestra institución en pacientes con COVID-19 severamente enfermos, los equipos de nefrología reportaban frecuentemente episodios de formación de coágulos dentro de las líneas y filtro de hemodiálisis, aun utilizando enoxaparina como anticoagulante. Esta observación empírica nos lleva a pensar que la tesis postulada por Ciceri puede ser correcta (4).

\section{Conclusión}

El SARS-CoV-2, agente etiológico del COVID-19, ha provocado la primera pandemia del siglo XXI y sus efectos han sido devastadores, tanto desde un punto de vista humano, por la pérdida de vidas humanas, como desde un punto de vista económico y social, con la pérdida de millones de empleos a nivel global y la amenaza de una subsecuente recesión económica. El hecho de que esta pandemia haya encontrado a todas las grandes potencias del mundo sin la capacidad de responder de una manera rápida y eficaz es señal de que se necesitan cambios estructurales en los sistemas globales de salud pública. Con esta recopilación de nuestras experiencias en Montefiore Health System esperamos poder contribuir al esfuerzo que realiza América Latina y, en especial, la República de Panamá para contener y mitigar los daños causados por esta pandemia. 


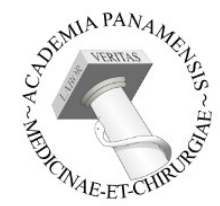

\section{Bibliografía}

1. Zhou P, Yang XL, Wang XG, Hu B, Zhang L, Zhang W, et al. A pneumonia outbreak associated with a new coronavirus of probable bat origin. Nature. 2020;579(7798):2703.

2. Silver AC, D. China is tightening its grip on coronavirus research 2020 [cited 2020 April 21]. Available from: https://www.nature.com/articles/d41586-020-01108-y.

3. Walls AC, Park YJ, Tortorici MA, Wall A, McGuire AT, Veesler D. Structure, Function, and Antigenicity of the SARS-CoV-2 Spike Glycoprotein. Cell. 2020;181(2):281-92.e6.

4. Ciceri F, Beretta L, Scandroglio AM, Colombo S, Landoni G, Ruggeri A, et al. Microvascular COVID-19 lung vessels obstructive thromboinflammatory syndrome (MicroCLOTS): an atypical acute respiratory distress syndrome working hypothesis. Crit Care Resusc. 2020.

5. Li H, Liu L, Zhang D, Xu J, Dai H, Tang N, et al. SARS-CoV-2 and viral sepsis: observations and hypotheses. Lancet. 2020.

6. Dolhnikoff M, Duarte-Neto AN, de Almeida Monteiro RA, Ferraz da Silva LF, Pierre de Oliveira E, Nascimento Saldiva PH, et al. Pathological evidence of pulmonary thrombotic phenomena in severe COVID-19. J Thromb Haemost. 2020.

7. Wu Z, McGoogan JM. Characteristics of and Important Lessons From the Coronavirus Disease 2019 (COVID-19) Outbreak in China: Summary of a Report of 72314 Cases From the Chinese Center for Disease Control and Prevention. Jama. 2020.

8. Xie J, Tong Z, Guan X, Du B, Qiu H. Clinical Characteristics of Patients Who Died of Coronavirus Disease 2019 in China. JAMA Network Open. 2020;3(4):e205619-e.

9. Li Q, Guan X, Wu P, Wang X, Zhou L, Tong Y, et al. Early Transmission Dynamics in Wuhan, China, of Novel Coronavirus-Infected Pneumonia. N Engl J Med. 2020;382(13):1199-207.

10. Zhou F, Yu T, Du R, Fan G, Liu Y, Liu Z, et al. Clinical course and risk factors for mortality of adult inpatients with COVID-19 in Wuhan, China: a retrospective cohort study. Lancet. 2020;395(10229):1054-62.

11. Richardson S, Hirsch JS, Narasimhan M, Crawford JM, McGinn T, Davidson KW, et al. Presenting Characteristics, Comorbidities, and Outcomes Among 5700 Patients Hospitalized With COVID-19 in the New York City Area. JAMA. 2020.

12. van Doremalen N, Bushmaker T, Morris DH, Holbrook MG, Gamble A, Williamson BN, et al. Aerosol and Surface Stability of SARS-CoV-2 as Compared with SARSCoV-1. N Engl J Med. 2020;382(16):1564-7.

13. Alhazzani W, Moller MH, Arabi YM, Loeb M, Gong MN, Fan E, et al. Surviving Sepsis Campaign: guidelines on the management of critically ill adults with Coronavirus Disease 2019 (COVID-19). Intensive Care Med. 2020.

14. Wu C, Chen X, Cai Y, Xia J, Zhou X, Xu S, et al. Risk Factors Associated With Acute Respiratory Distress Syndrome and Death in Patients With Coronavirus Disease 2019 Pneumonia in Wuhan, China. JAMA Intern Med. 2020. 


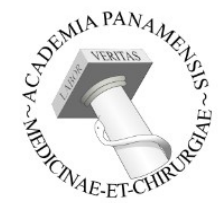

15. Shi S, Qin M, Shen B, Cai Y, Liu T, Yang F, et al. Association of Cardiac Injury With Mortality in Hospitalized Patients With COVID-19 in Wuhan, China. JAMA Cardiol. 2020.

16. Magagnoli J, Narendran S, Pereira F, Cummings T, Hardin JW, Sutton SS, et al. Outcomes of hydroxychloroquine usage in United States veterans hospitalized with Covid-19. medRxiv. 2020:2020.04.16.20065920.

17. Brower RG, Matthay MA, Morris A, Schoenfeld D, Thompson BT, Wheeler A. Ventilation with lower tidal volumes as compared with traditional tidal volumes for acute lung injury and the acute respiratory distress syndrome. $\mathrm{N}$ Engl $\mathrm{J}$ Med. 2000;342(18):1301-8.

18. Beun R, Kusadasi N, Sikma M, Westerink J, Huisman A. Thromboembolic events and apparent heparin resistance in patients infected with SARS-CoV-2. Int J Lab Hematol. 2020.

19. Barrett CD, Moore HB, Yaffe MB, Moore EE. ISTH interim guidance on recognition and management of coagulopathy in COVID-19: A Comment. Journal of Thrombosis and Haemostasis. 2020.

Publicación de la Revista Médica de Panamá. https://www.revistamedica.org

Manuscrito recibido: 23 de abril ,2020. Fecha de publicación temprana: 24 de abril, 2020.

DOI: http://dx.doi.org/10.37980/im.journal.rmdp.2020881

Editorial Infomedic International https://www.infomedicint.com

Acceso libre. Para uso individual; no comercial. Derechos reservados. 


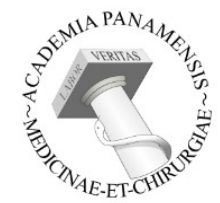

Figura $\mathrm{N}^{\mathbf{0}} 1$ - Total de casos de COVID-19 admitidos a Montefiore Health System (MHS), por fecha
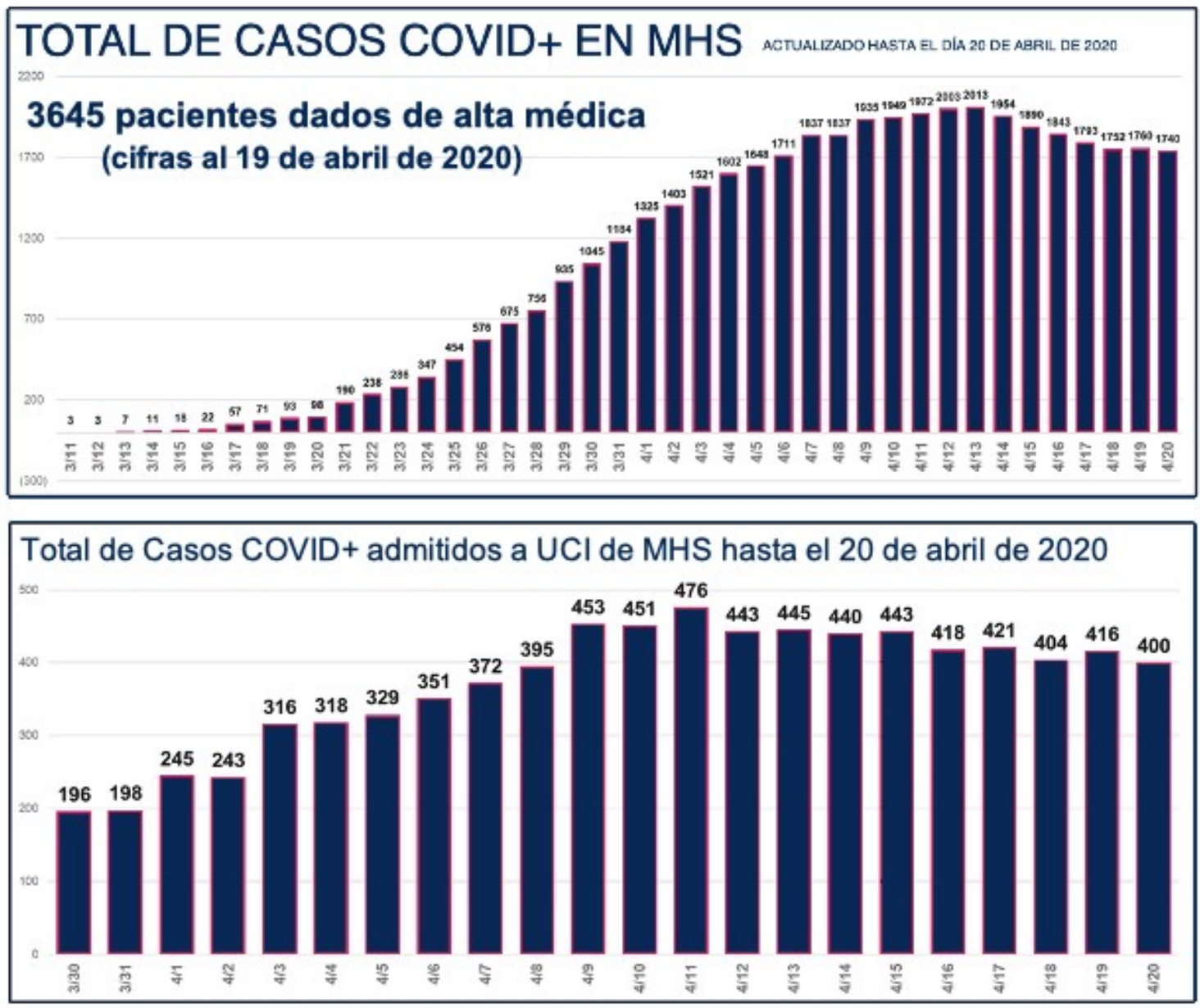

Leyenda: MHS: Montefiore Health System. UCI: Unidad de Cuidados Intensivos.

Publicación de la Revista Médica de Panamá. https://www.revistamedica.org

Manuscrito recibido: 23 de abril ,2020. Fecha de publicación temprana: 24 de abril, 2020.

DOI: http://dx.doi.org/10.37980/im.journal.rmdp.2020881

Editorial Infomedic International https://www.infomedicint.com

Acceso libre. Para uso individual; no comercial. Derechos reservados. 


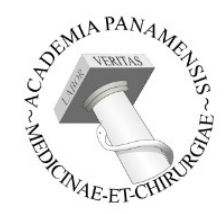

Figura No2 - Diagrama (no a escala) de la barrera utilizada para minimizar contacto físico durante la intubación de pacientes con COVID-19

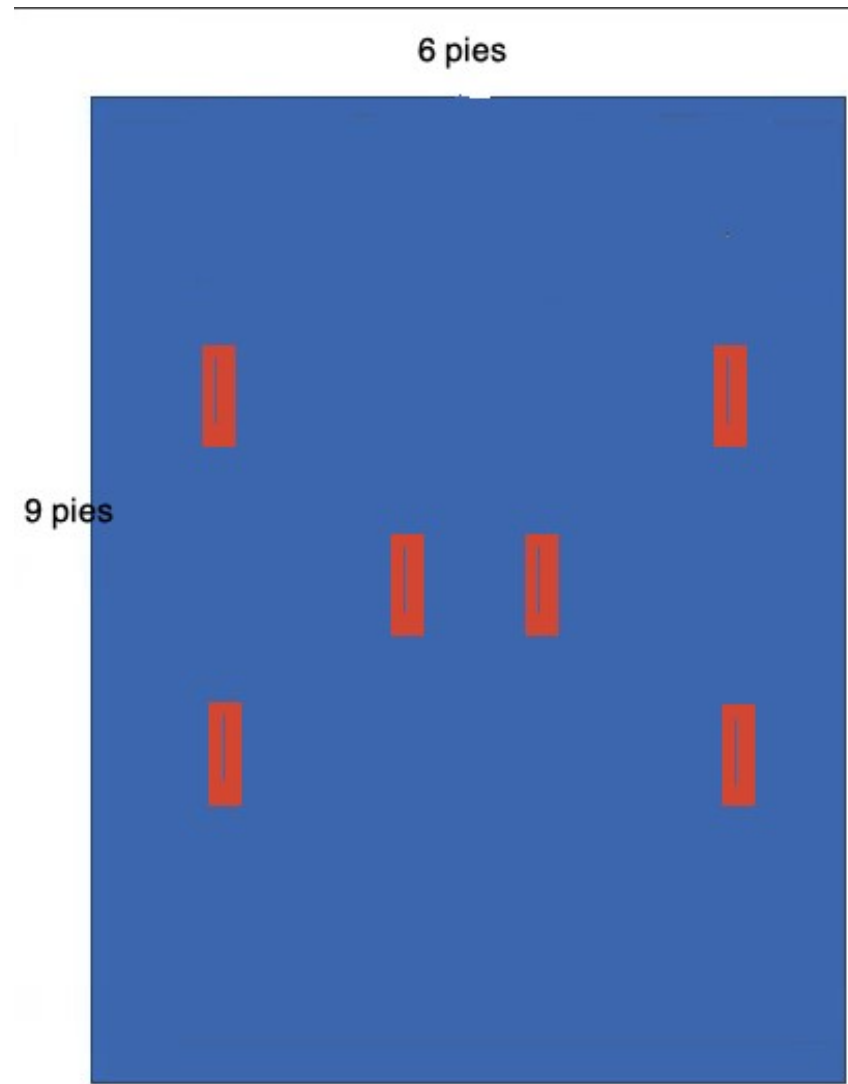

Publicación de la Revista Médica de Panamá. https://www.revistamedica.org

Manuscrito recibido: 23 de abril ,2020. Fecha de publicación temprana: 24 de abril, 2020.

DOI: http://dx.doi.org/10.37980/im.journal.rmdp.2020881

Editorial Infomedic International https://www.infomedicint.com

Acceso libre. Para uso individual; no comercial. Derechos reservados. 


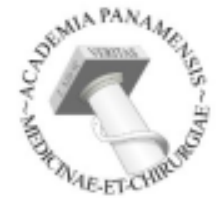

Figura N³ - Cardiomiopatía por COVID-19 presentándose como mímica de un infarto al miocardio con elevación del segmento ST (IAMEST).

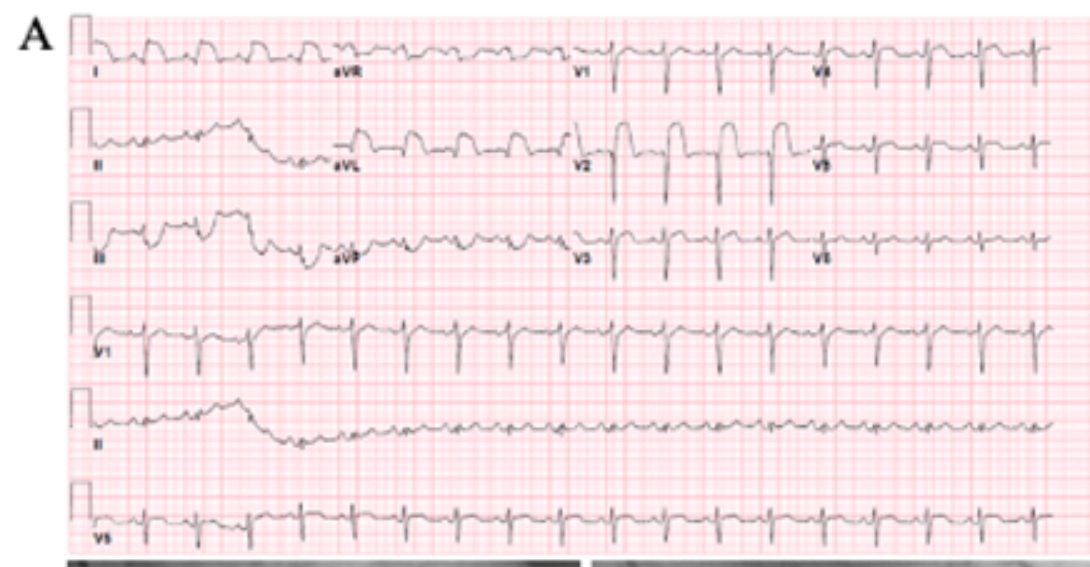

B
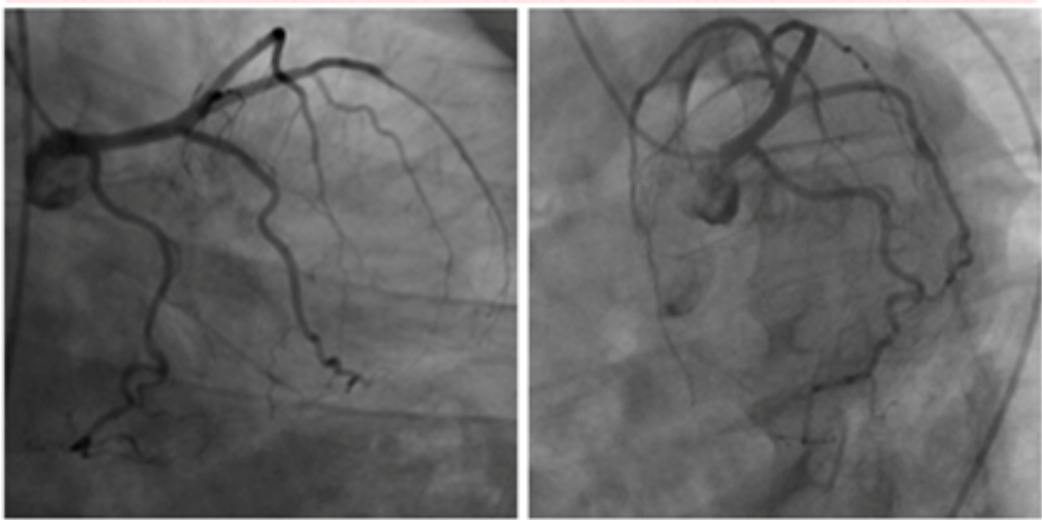

$\mathrm{C}$
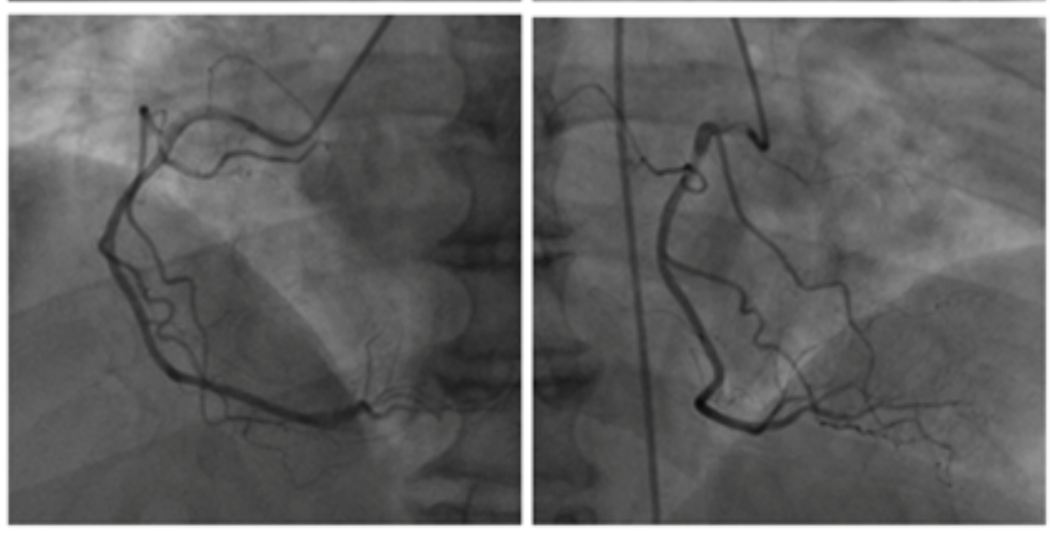

Publicación de la Revista Médica de Panamá. https://www.revistamedica.org

Manuscrito recibido: 23 de abril ,2020. Fecha de publicación temprana: 24 de abril, 2020.

DOI: $\underline{\text { http//dx.doi.org/10.37980/im.journal.rmdp. } 2020881}$

Editorial Infomedic International https://www.infomedicint.com

Acceso libre. Para uso individual; no comercial. Derechos reservados. 


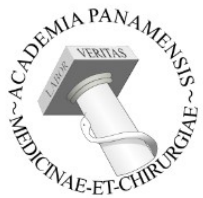

Podemos apreciar en A. Como el electrocardiograma muestra elevaciones del segmento ST sin una distribución vascular coronaria determinada B. La angiografía coronaria muestra el sistema coronario izquierdo intacto, sin ninguna lesión obstructiva. C. Hallazgos angiográficos similares en el sistema coronario derecho, donde tampoco se encuentra una lesión que explique las alteraciones electrocardiográficas. Este fue el paciente cero de Montefiore Health System.

Publicación de la Revista Médica de Panamá. https://www.revistamedica.org

Manuscrito recibido: 23 de abril ,2020. Fecha de publicación temprana: 24 de abril, 2020.

DOI: http://dx.doi.org/10.37980/im.journal.rmdp.2020881

Editorial Infomedic International https://www.infomedicint.com

Acceso libre. Para uso individual; no comercial. Derechos reservados. 